\title{
Molecular-orbital theory for the stopping power of atoms in the low velocity regime: the case of helium in alkali metals.
}

\author{
José J. Dorado and F. Flores. \\ Departamento de Física de la Materia Condensada C-XII, Facultad de Ciencias, \\ Universidad Autónoma de Madrid, E-28049 Madrid. Spain.
}

\begin{abstract}
A free-parameter linear-combination-of-atomic-orbitals approach is presented for analyzing the stopping power of slow ions moving in a metal. The method is applied to the case of He moving in alkali metals. Mean stopping powers for He present a good agreement with local density approximation calculations. Our results show important variations in the stopping power of channeled atoms with respect to their mean values.

34.50.Bw , 61.80.Mk
\end{abstract}

Typeset using REVTEX 


\section{INTRODUCTION}

The interaction of ions with condensed matter has drawn the attention of many researchers from the beginning of this century [1]. A great deal of work in this field has dealt with the energy loss of swift ions in solids. In this regard the work of Bethe [2], Fermi [3], Williams [4], and Lindhard [5] opened the modern way of calculating the stopping power of swift ions in condensed matter. The case of low-velocity projectiles is much more complicated due to the strong interaction of the moving ion with the solid. In this case, the projectile is dressed by a number of electrons that strongly screen the ion-solid interaction. Brandt [6] introduced a Thomas-Fermi statistical model in order to define an effective ion charge that takes into account how the bound electrons dress the projectile. Other researchers [7] have developed the Lindhard approach and have calculated the stopping power using a linear-response function. In recent developments [8,9], the stopping power for ions at low and intermediate velocities has been obtained by introducing the different electron-loss and -capture processes associated with the interaction of the projectile with the target 10 .

An important development in the calculation of the stopping power for very-low-velocity ions in solids appeared with the application of the local density theory to this field. Echenique, Nieminen, and Ritchie [11] calculated the stopping power for very slow ions moving in a uniform electron gas, using well-known techniques in this field. This approach has yielded a substantial improvement in the agreement between experimental data and theoretical calculations. The main limitation of this approach, as it has been used in the actual calculations, is the assumption of having an uniform electron gas in the solid. Although some attempts are currently tried to improve over this simplification [12,13, it could be convenient to try, at the same time, other alternatives that might be appropriate in the case of having very ionic or covalent solids.

The aim of the work presented in this paper is to apply to the stopping power field an approach recently developed for the calculation of electronic properties of solids [14,15] . This is a linear-combination-of-atomic-orbitals (LCAO) approach, whereby the electronic 
properties of the solids are calculated from the localized wave functions of the atoms of the solid. This approach tries to emphasize the local chemical properties of the solid and is deeply related to the work done by other groups, trying to calculate the stopping power for

ions in solids using the stopping power in the vapour target [16]. The advantage of these approaches is related to the non-uniformity of the target, since a local-density-approximation (LDA) calculation usually assumes a uniform electron gas inside a crystal. Thus the longterm aim of our approach is first, to calculate the stopping power for ions as a function of the ion position, in particular, near crystal surfaces; and secondly, to take into account the contribution of the different atomic orbitals of the target, mainly those orbitals which have such a localized size that can not be replaced by an uniform electron gas.

In this paper we have chosen to analyze the case of helium interacting with alkali metals. This is a case in which the interaction of the projectile and the target is simple. It is, however, a complicated system since it presents a long-range interaction between orbitals located at large separations. In these metals, the local density approach can be expected to be very good; therefore, we have chosen it as a strong test to the method we have developed, and the results obtained give strong support to it.

In the Secs. II and III, we present our model, the formalism used to solve it, and its application to the case of He in metals. In Sec. IV we discuss our results, and in Sec. V we present our conclusions.

\section{MODEL AND FORMALISM}

\section{A. General formalism}

Our model is an extension of a previous approach to the calculation of the electronic properties of the solids using a LCAO method [14,15]. The basic idea is to introduce the atomic orbitals $\psi_{\nu}, \nu=i, \alpha, i$ referring to the crystal site and $\alpha$ to a particular orbital, and the orthonormal basis $\phi_{\mu}$ 


$$
\phi_{\mu}=\sum_{\nu}\left(S^{-1 / 2}\right)_{\mu, \nu} \psi_{\nu}
$$

with

$$
S_{\nu \mu}=\left\langle\psi_{\nu} \mid \psi_{\mu}\right\rangle
$$

obtained using Löwdin's orthonormalization procedure [17]. Using this new basis, the electron Hamiltonian of a given system can be written in the following way:

$$
\begin{array}{r}
\hat{H}=\sum_{\nu, \sigma} E_{\nu}^{\sigma} \hat{n}_{\nu \sigma}+\sum_{\nu, \mu \neq \nu, \sigma} T_{\nu \mu}^{\sigma}\left(c_{\mu \sigma}^{\dagger} c_{\nu \sigma}+c_{\nu \sigma}^{+} c_{\mu \sigma}\right)+\sum_{\nu} U_{\nu}^{(0)} \hat{n}_{\nu \uparrow} \hat{n}_{\nu \downarrow} \\
+\frac{1}{2} \sum_{\nu, \mu \neq \nu, \sigma}\left[J_{\nu \mu}^{(0)} \hat{n}_{\mu \sigma} \hat{n}_{\nu-\sigma}+\left(J_{\nu \mu}^{(0)}-J_{x, \nu \mu}^{(0)}+J_{\nu \mu}^{(0)} S_{\nu \mu}^{2}\right) \hat{n}_{\mu \sigma} \hat{n}_{\nu \sigma}\right]
\end{array}
$$

with the operators $c_{\nu \sigma}^{\dagger}$ and $c_{\nu \sigma}$ related to the orthonormalized wave functions $\phi_{\nu}$. The different terms in Eq. (3) are analyzed in Refs. [14] and [15] ; here we only comment how to introduce the many-body terms of hamiltonian (3) in a one-body Hamiltonian by means of a Slater-like potential. This implies replacing Eq. (3) by the effective Hamiltonian:

$$
\hat{H}_{\mathrm{eff}}=\sum_{\nu, \sigma} \tilde{E}_{\nu}^{\sigma} \hat{n}_{\nu \sigma}+\sum_{\nu, \mu \neq \nu, \sigma} T_{\nu \mu}^{\sigma}\left(c_{\mu \sigma}^{+} c_{\nu \sigma}+c_{\nu \sigma}^{+} c_{\mu \sigma}\right)
$$

where

$$
\begin{aligned}
\tilde{E}_{\nu}^{\sigma}= & E_{\nu}^{\sigma}+U_{\nu}^{(0)}\left\langle\hat{n}_{\nu-\sigma}\right\rangle \\
& +\sum_{n u, \mu \neq \nu} J_{\nu \mu}^{(0)}\left\langle\hat{n}_{\mu-\sigma}\right\rangle+\sum_{n u, \mu \neq \nu}\left(J_{\nu \mu}^{(0)}-J_{x, \nu \mu}^{0}+J_{\nu \mu}^{(o)} S_{\nu \mu}^{2}+V_{\nu \mu}^{\mathrm{x}, \mathrm{c}}\right)\left\langle\hat{n}_{\mu \sigma}\right\rangle
\end{aligned}
$$

$V_{\mu}^{x, c}$ is the exchange and correlation potential [15] associated with the many-body terms of Eq. (3).

We start from Hamiltonian (田) and assume that its solution can be obtained in the static limit $v \rightarrow 0$, for the case of an atom moving inside a crystal (see Fig. 1). In our model, the different parameters of Hamiltonian (田), as well as its static solution, are calculated for a geometrical configuration, at each position of the external atom inside the crystal.

To proceed further, we assume that, due to the atomic motion, there is a time dependence of hamiltonian (4) through the ion velocity. This implies introducing a quasiadiabatic 
Hamiltonian, $\hat{H}_{\text {eff }}(t)$, with the different parameters, $E_{\nu}^{\sigma}$ and $T_{\nu \mu}$, having an explicit, but slowly, time dependence.

In order to calculate the stopping power at a given time and atomic position, the static solution of hamiltonian $\hat{H}_{\text {eff }}$ is introduced. This implies writing

$$
\hat{H}_{e f f}|n\rangle=E_{n}|n\rangle .
$$

Then, the stopping power (written as a function of the local time $t$, defining the projectile position) is given by the following equation [18]

$$
\frac{d E}{d t}=-2 \operatorname{Re} \sum_{n} \int_{-\infty}^{t} d t^{\prime} \frac{e^{-i w_{n 0}\left(t-t^{\prime}\right)}}{w_{n 0}}\left\langle 0\left|\frac{d \hat{H}_{\mathrm{eff}}(t)}{d t}\right| n\right\rangle\left\langle n\left|\frac{d \hat{H}_{\mathrm{eff}}\left(t^{\prime}\right)}{d t^{\prime}}\right| 0\right\rangle .
$$

[We are using atomic units $\left(\hbar=m=e^{-}=1\right)$.] Equation (7) is only valid in the quasiadiabatic limit, with the ion velocity going to zero. Notice that in Eq. (7), the eigenstates $|n\rangle$ correspond to the full Hamiltonian $\hat{H}_{\text {eff }}$, including the external ion, at the final time t. This approximation is obviously only appropriate for $v \rightarrow 0$.

Equation (7) can be further modified by noting that the dependence of $\hat{H}_{\text {eff }}$ with $\mathrm{t}$ appears through the coordinate $\mathbf{R}=\mathbf{R}_{0}+\mathbf{v} t$, of the external atom. Thus we write $\frac{d \hat{H}_{\text {eff }}(t)}{d t}=$ $(\mathbf{v} \cdot \nabla) \hat{H}_{\text {eff }}(\mathbf{R})$, and introduce the Fourier-transform $\hat{H}_{\text {eff }}(\mathbf{q})$ of $\hat{H}_{\text {eff }}(\mathbf{R})$. This yields

$$
\begin{aligned}
\frac{d E}{d t}= & -2 \operatorname{Re} \sum_{n} \int \frac{d \mathbf{q}}{(2 \pi)^{3}} \frac{d \mathbf{q}^{\prime}}{(2 \pi)^{3}} \int_{-\infty}^{t} \frac{e^{-i w_{n 0}\left(t-t^{\prime}\right)}}{w_{n 0}}(\mathbf{q} \cdot \mathbf{v})\left(\mathbf{q}^{\prime} \cdot \mathbf{v}\right) \\
& \times e^{i \mathbf{q} \cdot\left(\mathbf{R}_{0}+\mathbf{v} t\right)} e^{-i \mathbf{q}^{\prime} \cdot\left(\mathbf{R}_{0}+\mathbf{v} t^{\prime}\right)}\left\langle 0\left|\hat{H}_{e f f}(\mathbf{q})\right| n\right\rangle\left\langle n\left|\hat{H}_{e f f}\left(\mathbf{q}^{\prime}\right)\right| 0\right\rangle .
\end{aligned}
$$

This equation can be easily integrated on t'. Moreover, we introduce the one-electron eigenfunctions and eigenvalues, $|\mathbf{k}\rangle$, $\varepsilon_{k}$ of Hamiltonian $\hat{H}_{\text {eff }}$ in Eq. (8) to define $|n\rangle$ and $w_{n 0}$.

These steps yield the following results:

$$
\begin{aligned}
\frac{d E}{d t}= & 4 \pi \sum_{k\left\langle k_{F}, k^{\prime}\right\rangle k_{F}} \int \frac{d \mathbf{q}}{(2 \pi)^{3}} \int \frac{d \mathbf{q}^{\prime}}{(2 \pi)^{3}} \frac{(\mathbf{q} \cdot \mathbf{v})\left(\mathbf{q}^{\prime} \cdot \mathbf{v}\right)}{w_{k k^{\prime}}} \\
& \times\left\langle\mathbf{k}^{\prime}\left|\hat{H}_{\mathrm{eff}}(\mathbf{q}) e^{i \mathbf{q} \cdot \mathbf{R}}\right| \mathbf{k}\right\rangle\left\langle\mathbf{k}\left|\hat{H}_{\mathrm{eff}}(\mathbf{q}) e^{-i \mathbf{q}^{\prime} \cdot \mathbf{R}}\right| \mathbf{k}^{\prime}\right\rangle \\
& \times \delta\left(w_{k k^{\prime}}+\mathbf{q} \cdot \mathbf{v}\right),
\end{aligned}
$$

where the spin has been added up and $w_{k k^{\prime}}=\varepsilon_{k^{\prime}}-\varepsilon_{k}$. Note that $|\mathbf{k}\rangle$ and $\varepsilon_{k}$ are the eigenfunctions and eigenvalues of the total Hamiltonian, $\hat{H}_{\text {eff }}(\mathbf{R})$, with the external ion 
included. One should remember, however, that $|\mathbf{k}\rangle$ and $\left|\mathbf{k}^{\prime}\right\rangle$ are not eigenfunctions of $\hat{H}_{\mathrm{eff}}(\mathbf{q}):$

$$
\hat{H}_{\mathrm{eff}}(\mathbf{q})=\int d \mathbf{R}^{\prime} e^{-i \mathbf{q} \cdot \mathbf{R}^{\prime}} \hat{H}_{\mathrm{eff}}\left(\mathbf{R}^{\prime}\right)
$$

It is of interest to make contact between Eq. (9) and the linear-response theory. In this case, the total Hamiltonian is written as the sum of the unperturbed hamiltonian $\hat{H}_{0}$ and a perturbation $\hat{H}_{\text {pert }}=\hat{V}$. Then, Eq. (9) can be transformed by taking for $|\mathbf{k}\rangle$ and $\left|\mathbf{k}^{\prime}\right\rangle$, the eigenfunctions of $\hat{H}_{0}$; moreover, the perturbation $\hat{V}$, can be written as follows:

$$
\hat{V}=\int d \mathbf{r} \frac{Z}{|\mathbf{R}-\mathbf{r}|} \hat{\rho}(\mathbf{r})
$$

where $Z$ is the external ion charge and $\mathbf{R}$ its position. Then, the power loss is given by the following equation (linear theory):

$$
\begin{aligned}
\frac{d E}{d t}= & 4 \pi \sum_{\substack{k\left\langle k_{F}, k^{\prime}\right\rangle k_{F}\\
}} \int \frac{d \mathbf{q}}{(2 \pi)^{3}} \int \frac{d \mathbf{q}^{\prime}}{(2 \pi)^{3}}\left(\frac{4 \pi Z}{q^{2}}\right)\left(\frac{4 \pi Z}{q^{\prime 2}}\right)(\mathbf{q} \cdot \mathbf{v}) \\
& \times e^{i\left(\mathbf{q}-\mathbf{q}^{\prime}\right) \cdot \mathbf{R}}\left\langle\mathbf{k}^{\prime}\left|\rho^{+}(\mathbf{q})\right| \mathbf{k}\right\rangle\left\langle\mathbf{k}\left|\rho\left(\mathbf{q}^{\prime}\right)\right| \mathbf{k}^{\prime}\right\rangle \delta\left(w_{k k^{\prime}}+\mathbf{q} \cdot \mathbf{v}\right)
\end{aligned}
$$

or, equivalently,

$$
\begin{aligned}
\frac{d E}{d t}= & 2 \int \frac{d \mathbf{q}}{(2 \pi)^{3}} \int d \mathbf{q}^{\prime}\left(\frac{4 \pi Z}{q^{2}}\right)\left(\frac{4 \pi Z}{q^{\prime 2}}\right)(\mathbf{q} \cdot \mathbf{v}) \\
& \times e^{i\left(\mathbf{q}-\mathbf{q}^{\prime}\right) \cdot \mathbf{R}} \operatorname{Im} \chi\left(\mathbf{q}, \mathbf{q}^{\prime} ;-\mathbf{q} \cdot \mathbf{v}\right),
\end{aligned}
$$

where $\operatorname{Im} \chi\left(\mathbf{q}, \mathbf{q}^{\prime} ; w\right)$ is the metal polarizability.

For an homogeneous system, only $\mathbf{q}=\mathbf{q}^{\prime}$ contributes, and Eq. (13) yields

$$
\frac{d E}{d t}=2 \int \frac{d \mathbf{q}}{(2 \pi)^{3}}\left(\frac{4 \pi Z}{q^{2}}\right)^{2}(\mathbf{q} \cdot \mathbf{v}) \operatorname{Im} \chi(\mathbf{q} ;-\mathbf{q} \cdot \mathbf{v})
$$

in agreement with other Refs. [5, [18]. Equation (9) is the basic equation giving the stopping power of the moving ion, in the low velocity limit, within our LCAO approach. In Eq. (9) the critical quantity to calculate, using the static interaction between the external charge and the solid, is $\left\langle\mathbf{k}\left|\hat{H}_{\mathrm{eff}}(\mathbf{q})\right| \mathbf{k}^{\prime}\right\rangle$. In this paper we shall concentrate on the He case; this provides a simple case in which to test the method discussed here. 


\section{B. Static interaction of He with a metal}

In this section, we will present a summary of the main results discussed in Ref. [14]. We shall also extend this discussion in order to calculate the matrix elements $\left\langle\mathbf{k}\left|\hat{H}_{\mathrm{eff}}(\mathbf{q})\right| \mathbf{k}^{\prime}\right\rangle$, needed for the calculation of the stopping power. Following Ref. [14], we start by considering the one-electron interactions between the He $1 s$ level and a metal band that is represented in Fig. 2 by a half-occupied $s$ level. As discussed in Ref. [14, there are two different oneelectron interactions. First, due to the overlap $S$ between the He $1 s$ wave function and the metal orbital $\left(S=\left\langle\psi_{M} \mid \psi_{\mathrm{He}}\right\rangle\right)$, there is an increase in the kinetic energy of the electrons of the system. This is measured by the following shift of the one-electron terms:

$$
\begin{gathered}
\delta E_{M}^{(1)}=\frac{1}{4} S^{2}\left(E_{M}^{0}-E_{\mathrm{He}}^{0}\right)-S T, \\
\delta E_{\mathrm{He}}^{(1)}=-\frac{1}{4} S^{2}\left(E_{M}^{0}-E_{\mathrm{He}}^{0}\right)-S T,
\end{gathered}
$$

where $T$, the hopping between the two orbitals, $\psi_{M}$ and $\psi_{\mathrm{He}}$, is found to be $-\frac{1}{2} S\left(E_{M}^{0}-E_{\mathrm{He}}^{0}\right)$. $E_{M}^{0}$ and $E_{\mathrm{He}}^{0}$ are the metal and He energy levels. Second, due to the hopping $T$ between the two orbitals we find a hybridization contribution to the total energy given by the following shift in $E_{M}^{0}$ and $E_{\mathrm{He}}^{0}$ :

$$
\begin{gathered}
\delta E_{M}^{(2)}=\frac{T^{2}}{\left(E_{M}-E_{\mathrm{He}}\right)}, \\
\delta E_{\mathrm{He}}^{(2)}=-\frac{T^{2}}{\left(E_{M}-E_{\mathrm{He}}\right)} .
\end{gathered}
$$

Combining Eqs. (15)-(18), we find the following contributions:

$$
\begin{gathered}
\delta E_{M}=S^{2}\left(E_{M}-E_{\mathrm{He}}\right) \\
\delta E_{\mathrm{He}}=0
\end{gathered}
$$

These shifts in the one-electron levels yield the following contribution to the repulsive energy: 


$$
\delta V_{\text {repulsive }}^{\text {one-electron }}=n_{M} S^{2}\left(E_{M}-E_{\mathrm{He}}\right),
$$

where $n_{M}$ is the number of electrons in the metal orbital.

Many-body contributions have also been discussed in Ref. [14. These terms can be written in a way similar to Eq. (21); in Ref. [14] it was found that the total repulsive energy between the metal atom and He is given by

$$
\delta V_{\text {repulsive }}^{\text {one-electron }}=n_{M} S^{2}\left(E_{M}-E_{\mathrm{He}}\right)+n_{M}\left(-J_{x}^{0}+S^{2} J_{0}\right)+V_{\text {electrostatic }}
$$

where $J_{x}^{0}$ is the exchange integral between the metal and the He orbitals, $J_{0}$ the Coulomb interaction between the same orbitals, and $V_{\text {electrostaric }}$ the electrostatic interaction between the total charges of the two atoms. For a He-orbital going like $\left(\frac{\beta^{3}}{\pi}\right)^{1 / 2} e^{-\beta r}$ we find that

$$
-J_{x}^{0}+V_{\text {electrostatic }}=-\frac{3}{8} \beta S^{2}
$$

This shows that the repulsive potential can be written as follows:

$$
V_{\text {repulsive }}=n_{M} S^{2}\left(E_{M}-E_{\mathrm{He}}-\frac{3}{8} \beta+J_{0}\right)
$$

In our actual problem we are interested in calculating $\left\langle\mathbf{k}^{\prime}\left|\hat{H}_{\text {eff }}(\mathbf{R})\right| \mathbf{k}\right\rangle$, the matrix element of the total Hamiltonian between the one-electron states $|\mathbf{k}\rangle$. We will show how Eq. (24) can be related to $\left\langle\mathbf{k}^{\prime}\left|\hat{H}_{\text {eff }}(\mathbf{R})\right| \mathbf{k}\right\rangle$. To this end, we start by discussing the solution of the total Hamiltonian (crystal plus the external atom) within a one-electron approximation. The solution of this Hamiltonian $\hat{H}$ is given by

$$
\psi=\sum_{k} c_{k} \psi_{k}+c_{\mathrm{He}} \psi_{\mathrm{He}}
$$

where $\psi_{k}$ are the eigenfunctions of the crystal Hamiltonian, $\hat{H}_{0}$, and $\psi_{\mathrm{He}}$ the 1 s orbital of He. In writing Eq. (25), we assume that the total Hamiltonian (in our one-electron approximation) is given by $\hat{H}=\hat{H}_{0}+\hat{V}_{\mathrm{He}}$, where $\hat{V}_{\mathrm{He}}$ defines the one-electron potential created by the atom. The eigenvalues and the eigenfunctions of $\hat{H}$ are given by the secular equation 


$$
\operatorname{det}\left|\left\langle\psi_{i}|-E+\hat{H}| \psi_{j}\right\rangle\right|=0 \text {. }
$$

Now, we follow Ref. [14] and introduce the orthonormalized wave functions [as done in Eq. (1) for the basis $\left.\psi_{\nu}\right]$

$$
\phi_{i}=\sum_{i^{\prime}}\left(S^{-1 / 2}\right)_{i i^{\prime}} \psi_{i^{\prime}}
$$

with

$$
S_{k \mathrm{He}}=\left\langle\psi_{k} \mid \psi_{\mathrm{He}}\right\rangle
$$

and

$$
S_{k k^{\prime}}=\left\langle\psi_{k} \mid \psi_{k^{\prime}}\right\rangle=0
$$

Using Eq. (27) we define the following effective hamiltonian

$$
\hat{H}_{\text {eff }}=S^{-1 / 2} \hat{H} S^{-1 / 2} \text {. }
$$

In Ref. [14], the diagonal terms of the effective Hamiltonian were calculated up to second order in the overlap, a small parameter used for calculating $S^{-1 / 2}$ in a series expansion, while the off-diagonal terms were only obtained up to first order. In our actual problem we need to calculate $\left(\hat{H}_{\text {eff }}\right)_{k k^{\prime}}$ up to second order in the overlap, the smallest surviving term of the expansion.

Proceeding in this way, we obtain the following results:

$$
\begin{aligned}
\left(\hat{H}_{\mathrm{eff}}\right)_{k \mathrm{He}}= & T_{k \mathrm{He}}=-\frac{1}{2} S_{k \mathrm{He}}\left(E_{k}^{0}-E_{\mathrm{He}}^{0}\right) \\
\left(\hat{H}_{\mathrm{eff}}\right)_{k k^{\prime}}=T_{k k^{\prime}}= & \left(V_{\mathrm{He}}\right)_{k k^{\prime}}-\frac{1}{2}\left(T_{k \mathrm{He}} S_{\mathrm{He} k^{\prime}}+T_{k^{\prime} \mathrm{He}} S_{\mathrm{He} k}\right) \\
& +\frac{1}{4}\left(\frac{E_{k}^{0}+E_{k^{\prime}}^{0}}{2}-E_{\mathrm{He}}\right) S_{\mathrm{He} k^{\prime}} S_{\mathrm{He} k}
\end{aligned}
$$

where $E_{k}^{0}$ and $E_{\mathrm{He}}^{0}$ are the k-state and the atomic levels, respectively.

Equation (31) was already discussed in Ref. [14], and found to be valid for a very localized wavefunction like the He $1 s$ level. Equation (32) is the new equation we are looking for; here 
$\left(V_{H e}\right)_{k k^{\prime}}$ is associated with the direct perturbation introduced by the He atom on the metal. This perturbation is basically due to the atomic Hartree potential, and to the exchange perturbation created by the He $1 s$ level.

Equations (31) and (32) can be further approximated by taking $E_{k}^{0}$, the one-electron $k$ state levels, equal to $E_{M}^{0}$ a mean level of the metal band (notice that the He level $E_{\mathrm{He}}^{0}$ is very deep and that replacing $E_{k}^{0}$ by $E_{M}^{0}$ is a good approximation) . Then Eqs. (31) and (32) read

$$
\begin{gathered}
T_{k \mathrm{He}}=-\frac{1}{2} S_{k \mathrm{He}}\left(E_{M}-E_{\mathrm{He}}\right) \\
T_{k k^{\prime}}=\left(V_{\mathrm{He}}\right)_{k k^{\prime}}-\frac{1}{2}\left(T_{k \mathrm{He}} S_{\mathrm{He} k^{\prime}}+T_{k^{\prime} \mathrm{He}} S_{\mathrm{Hek}}\right)+\frac{1}{4}\left(E_{M}-E_{\mathrm{He}}\right) S_{\mathrm{He} k^{\prime}} S_{\mathrm{He} k} \\
=\left(V_{\mathrm{He}}\right)_{k k^{\prime}}+\frac{3}{4}\left(E_{M}-E_{\mathrm{He}}\right) S_{\mathrm{He} k^{\prime}} S_{\mathrm{He} k} .
\end{gathered}
$$

The terms appearing in Eq. (34), that depend on $T_{k \mathrm{He}}$ and $S_{k \mathrm{He}}$, are equivalent to the ones going like $(-S T)$, in Eq. (15), if $T$ is replaced here by $-\frac{1}{2} S\left(E_{M}-E_{\mathrm{He}}\right)$; this shows how the one-electron correction to the metal level $\frac{3}{4} S^{2}\left(E_{M}-E_{\mathrm{He}}\right)$ coincides with the one-electron contribution to the off-diagonal term in $T_{k k^{\prime}}$ if $S^{2}$ is replaced by $S_{k \mathrm{He}} S_{\mathrm{He} k^{\prime}}$.

Returning to Eq. (33), we should comment that $T_{k \mathrm{He}}$ is a first order term in the overlap $S_{\mathrm{Hek}}$ while $T_{k k^{\prime}}$ is second order $\left[\left(V_{\mathrm{He}}\right)_{k k^{\prime}}\right.$ included]. The first order term $T_{k \mathrm{He}}$ introduces an effective second order contribution to $T_{k k^{\prime}}$ given by

$$
\frac{T_{k \mathrm{He}} T_{\mathrm{He} k^{\prime}}}{E_{M}^{0}-E_{\mathrm{He}}^{0}} .
$$

Combining Eqs. (33) and (34) with Eq. (35) we get the following effective interaction:

$$
T_{k k^{\prime}}=\left(V_{\mathrm{He}}\right)_{k k^{\prime}}+\left(E_{M}^{0}-E_{\mathrm{He}}^{0}\right) S_{k \mathrm{He}} S_{\mathrm{He} k^{\prime}} .
$$

This is the one-electron contribution to the effective hopping between the crystal wave functions $|\mathbf{k}\rangle$ and $\left|\mathbf{k}^{\prime}\right\rangle$, as induced by the external atom. When the crystal wavefunctions $|\mathbf{k}\rangle$ are developed in a local basis

$$
\mid \mathbf{k}>=\sum_{i} c_{i}(\mathbf{k}) \phi_{i}
$$


$\phi_{i}$ being the orthonormalized wave functions associated with the metal atom , Eq. (36) reads as follows:

$$
T_{i i^{\prime}}=\left(V_{\mathrm{He}}\right)_{i i^{\prime}}+\left(E_{M}^{0}-E_{\mathrm{He}}^{0}\right) S_{i \mathrm{He}} S_{\mathrm{He} i^{\prime}}
$$

Equation (38) is the fundamental equation making contact between the repulsive potential given by Eq. (19) and $T_{i i^{\prime}}$. Many-body contributions are partially taken into account in Eq. (38) by means of the term $\left(V_{H e}\right)_{i i^{\prime}}$ which includes the bare Hartree and bare exchange contributions, equivalent to $V_{\text {electrostatic }}$ and $-J_{x}^{0}$ in Eq. (22). The extra term $S^{2} J_{0}$, appearing in Eq. (22) is due to the effect of the overlap between the $|\mathbf{k}\rangle$ and He orbitals in the total exchange interaction.

This discussion and the results of Eq. (38) suggest to introduce the following effective interaction between the $i$ and $i^{\prime}$ orbitals

$$
\left(T_{\mathrm{eff}}\right)_{i i^{\prime}}=S_{i \mathrm{He}} S_{\mathrm{He} i^{\prime}}\left(E_{M}^{0}-E_{\mathrm{He}}^{0}-\frac{3}{8} \beta+\left\langle J^{0}\right\rangle\right)
$$

This equation should be compared with Eq. (24) that yields the total repulsive potential between He and the metal atoms.

In this equation, $\left\langle J^{0}\right\rangle$ is associated with the effect of the overlap between the He $1 s$ orbital and the atomic wave functions of the metal in the exchange interaction created by the He-orbital. In Eq. (24), $J^{0}$ is the Coulomb interaction between the atomic wavefunction and the He $1 s$ orbital; in Eq. (39) we have introduced $\left\langle J^{0}\right\rangle$, the mean value of this Coulomb interaction in the crystal unit cell (the change of $J^{0}$ along this unit cell is small, less than $10 \%)$

Equation (39) is the main equation giving the effective matrix elements creating the excitation between the $i$ and $i^{\prime}$ orbitals, or the Bloch wave functions $|\mathbf{k}\rangle$ and $\left|\mathbf{k}^{\prime}\right\rangle$ in the crystal, in this basis:

$$
\left(T_{\mathrm{eff}}\right)_{k k^{\prime}}=S_{k \mathrm{He}} S_{\mathrm{He} k^{\prime}}\left(E_{M}^{0}-E_{\mathrm{He}}^{0}-\frac{3}{8} \beta+\left\langle J^{0}\right\rangle\right)
$$




\section{DYNAMIC INTERACTION OF HELIUM WITH A METAL}

Once we have obtained the static interaction of He with the metal, and the effective matrix elements, we will discuss how to combine this result with the general Eq. (9) to calculate the stopping power for He. First of all, let us mention that we shall use Eq. (9) by assuming that $|\mathbf{k}\rangle$ and $\left|\mathbf{k}^{\prime}\right\rangle$ are well described, for the He case, by the unperturbed crystal wave functions. This is a good approximation in our current case due to the small overlap between the He $1 s$ and the localized metal wave functions.

Then, the starting point is the equation

$$
\left[\hat{T}_{\text {eff }}(\mathbf{R})\right]_{k k^{\prime}}=V_{0} S_{k \mathrm{He}} S_{\mathrm{He} k^{\prime}}
$$

where

$$
V_{0}=\left(E_{M}-E_{\mathrm{He}}-\frac{3}{8} \beta+\left\langle J^{0}\right\rangle\right)
$$

The overlap between the $1 s$ He state and the $|\mathbf{k}\rangle$ wave functions is written in the following way

$$
\left\langle\mathbf{k} \mid \psi_{\mathrm{He}}\right\rangle=\int d \mathbf{r} \psi_{k}^{*}(\mathbf{r}) \psi_{\mathrm{He}}(\mathbf{r}) \simeq \psi_{k}^{*}\left(\mathbf{R}_{\mathrm{He}}\right) \int d \mathbf{r} \psi_{\mathrm{He}}(\mathbf{r})
$$

where we replace $\psi_{k}^{*}(\mathbf{r})$ by $\psi_{k}^{*}\left(\mathbf{R}_{\mathrm{He}}\right)$, assuming the He $1 s$ level to be very localized. This allows us to write:

$$
\begin{aligned}
\left\langle\mathbf{k}\left|\hat{H}_{\mathrm{eff}}(\mathbf{R})\right| \mathbf{k}^{\prime}\right\rangle & =V_{0} \psi_{k}^{*}\left(\mathbf{R}_{\mathrm{He}}\right) \psi_{k^{\prime}}\left(\mathbf{R}_{\mathrm{He}}\right)\left[\int d \mathbf{r} \psi_{\mathrm{He}}(\mathbf{r})\right]^{2} \\
& =V_{0}^{\prime} \psi_{k}^{*}\left(\mathbf{R}_{\mathrm{He}}\right) \psi_{k^{\prime}}\left(\mathbf{R}_{\mathrm{He}}\right) .
\end{aligned}
$$

This yields [see Eqs. (9), (10)]

$$
\begin{aligned}
H_{\mathrm{eff}}(\mathbf{q}) & =V_{0}^{\prime} \int d \mathbf{R} e^{i \mathbf{q} \cdot \mathbf{R}} \psi_{k}^{*}(\mathbf{R}) \psi_{k^{\prime}}(\mathbf{R}) \\
& =V_{0}^{\prime} I_{k k^{\prime}}(\mathbf{q})
\end{aligned}
$$

and 


$$
\begin{aligned}
\frac{d E}{d t}= & 4 \pi \sum_{k\left\langle k_{F}, k^{\prime}\right\rangle k_{F}}\left(V_{0}^{\prime}\right)^{2} \int \frac{d \mathbf{q}}{(2 \pi)^{3}} \int \frac{d \mathbf{q}^{\prime}}{(2 \pi)^{3}} \frac{(\mathbf{q} \cdot \mathbf{v})\left(\mathbf{q}^{\prime} \cdot \mathbf{v}\right)}{w_{k k^{\prime}}} \\
& \times I_{k k^{\prime}}(\mathbf{q}) I_{k^{\prime} k}\left(\mathbf{q}^{\prime}\right) e^{i\left(\mathbf{q}-\mathbf{q}^{\prime}\right) \cdot \mathbf{R}} \delta\left(w_{k k^{\prime}}+\mathbf{q} \cdot \mathbf{v}\right) .
\end{aligned}
$$

This is the general equation giving the power loss at a given point $\mathbf{R}$. Notice that due to the crystal simmetry $\mathbf{k}^{\prime}=\mathbf{k}-\mathbf{q}$ and $\mathbf{q}^{\prime}=\mathbf{q}-\mathbf{G}, \mathbf{G}$ being a crystal reciprocal vector.

If we are only interested in the mean power loss and neglect the $\mathbf{R}$ dependence, we should concentrate on the $\mathbf{q}=\mathbf{q}^{\prime}$ contribution. Then Eq. (46) yields

$$
\begin{aligned}
\frac{d E}{d t}= & 4 \pi\left(V_{0}^{\prime}\right)^{2} \int_{-\infty}^{\infty} \frac{d \mathbf{k}}{(2 \pi)^{3}} \int_{-\infty}^{\infty} \frac{d \mathbf{q}}{(2 \pi)^{3}}(\mathbf{q} \cdot \mathbf{v}) \Theta\left(k_{F}-k\right) \Theta\left(k^{\prime}-k_{F}\right) \\
& \times I_{k k^{\prime}}(\mathbf{q}) I_{k^{\prime} k}(\mathbf{q}) \delta\left(w_{k k^{\prime}}+\mathbf{q} \cdot \mathbf{v}\right),
\end{aligned}
$$

with $\mathbf{k}^{\prime}=\mathbf{k}-\mathbf{q}$ and $\Theta$ is the step function.

Equation (47) depends on the velocity direction of the projectile. As we shall only consider the case of He moving in alkali metals, crystals that have a very small anisotropy, we shall calculate the stopping power by taking an average on all the $\mathbf{v}$ directions, which will enable us to compare our results with other works [11]. Then:

$$
\frac{1}{v} \frac{d E}{d x}=\frac{\int_{-1}^{1} d \cos \theta_{v} d E / d t}{2 v^{2}}
$$

which redefines $d E / d x$.

Equation (47) is our fundamental equation for calculating the stopping power for He, in the low-velocity limit. This equation can be written in a local basis by developing the $\mathbf{k}$ states in the atomic orbitals of the crystal. In general, we shall assume that the metal wavefunctions are given by an effective one-electron Hamiltonian $\hat{H}_{0}$, such that

$$
\hat{H}_{0}|\mathbf{k}\rangle=E(\mathbf{k})|\mathbf{k}\rangle \text {. }
$$

Then, the solution of this hamiltonian yields

$$
|\mathbf{k}\rangle=\sum_{i, \alpha} c_{\alpha}(k) e^{i \mathbf{k} \cdot \mathbf{R}_{i}} \phi_{i \alpha}\left(\mathbf{r}-\mathbf{R}_{i}\right)
$$

where $\phi_{i \alpha}\left(\mathbf{r}-\mathbf{R}_{i}\right)$ are the orthonormalized wave functions associated with the $i$ site $(\alpha$ measures the number of orbitals per site). On the other hand $\phi_{i \alpha}\left(\mathbf{r}-\mathbf{R}_{i}\right)$ should be expressed 
as a function of the localized atomic orbitals $\psi_{i \alpha}\left(\mathbf{r}-\mathbf{R}_{i}\right)$ using Eq. (1). By substituting Eqs. (50) and (11) into Eq. (47), we find the following result

$$
\begin{aligned}
\frac{1}{v} \frac{d E}{d x}= & 2 \pi\left(V_{0}^{\prime}\right)^{2} \int_{-1}^{1} d \cos \theta_{v} \int_{-\infty}^{\infty} \frac{d \mathbf{k}}{(2 \pi)^{3}} \int_{-\infty}^{\infty} \frac{d \mathbf{q}}{(2 \pi)^{3}} \frac{(\mathbf{q} \cdot \mathbf{v})}{v^{2}} \Theta\left(k_{F}-k\right) \Theta\left(k^{\prime}-k_{F}\right) \\
& \times \sum_{\mathbf{R}_{1}, \mathbf{R}_{2}} \sum_{\alpha, \beta, \gamma, \delta} c_{\alpha}^{*}(\mathbf{k}) c_{\gamma}(\mathbf{k}) c_{\beta}^{*}\left(\mathbf{k}^{\prime}\right) c_{\delta}\left(\mathbf{k}^{\prime}\right) \\
& \times \sum_{\alpha^{\prime}, \beta^{\prime}}\left(S(\mathbf{k})^{-1 / 2}\right)_{\alpha \alpha^{\prime}} I_{\alpha^{\prime} \beta^{\prime}}^{\mathbf{R}_{1}}(\mathbf{q})\left(S(\mathbf{k})^{-1 / 2}\right)_{\beta \beta^{\prime}} \\
& \times \sum_{\gamma^{\prime}, \delta^{\prime}}\left(S\left(\mathbf{k}^{\prime}\right)^{-1 / 2}\right)_{\gamma \gamma^{\prime}} I_{\gamma^{\prime} \delta^{\prime}}^{\mathbf{R}_{2}}(\mathbf{q})\left(S\left(\mathbf{k}^{\prime}\right)^{-1 / 2}\right)_{\delta \delta^{\prime}} \\
& \times e^{i(\mathbf{k}-\mathbf{q}) \cdot\left(\mathbf{R}_{1}-\mathbf{R}_{2}\right)} \delta\left(w_{k k^{\prime}}+\mathbf{q} \cdot \mathbf{v}\right),
\end{aligned}
$$

where

$$
\begin{gathered}
I_{\beta \gamma}^{\mathbf{R}_{1}}(\mathbf{q})=\int d \mathbf{r} e^{i \mathbf{q} \cdot \mathbf{r}} \psi_{\beta}(\mathbf{r}) \psi_{\gamma}\left(\mathbf{r}-\mathbf{R}_{1}\right), \\
\left(S(\mathbf{k})^{-1 / 2}\right)_{\alpha \beta}=\sum_{\mathbf{R}} e^{i \mathbf{k} \cdot \mathbf{R}}\left(S^{-1 / 2}(\mathbf{R})\right)_{\alpha \beta}, \\
S(\mathbf{k})_{\alpha \beta}=\sum_{\mathbf{R}} e^{i \mathbf{k} \cdot \mathbf{R}} \int d \mathbf{r} \psi_{\alpha}(\mathbf{r}) \psi_{\beta}(\mathbf{r}-\mathbf{R}) .
\end{gathered}
$$

Finally, we relate $c_{\beta}^{*}(\mathbf{k}) c_{\alpha}(\mathbf{k})$ to the metal Green functions $G_{\beta \alpha}(\mathbf{k}, w)$ by the equations

$$
\begin{gathered}
\Theta\left(k_{F}-k\right) c_{\beta}(\mathbf{k}) c_{\alpha}^{*}(\mathbf{k})=\frac{1}{\pi} \int_{-\infty}^{E_{F}} d w \operatorname{Im} G_{\beta \alpha}(\mathbf{k}, w) \\
\Theta\left(k^{\prime}-k_{F}\right) c_{\delta}^{*}(\mathbf{k}) c_{\gamma}(\mathbf{k})=-\frac{1}{\pi} \int_{E_{F}}^{\infty} d w \operatorname{Im} G_{\delta \gamma}(\mathbf{k}, w) .
\end{gathered}
$$

This yields

$$
\begin{aligned}
\frac{1}{v} \frac{d E}{d x}= & 2 \pi\left(V_{0}^{\prime}\right)^{2} \int_{-1}^{1} d \cos \theta_{v} \int_{-\infty}^{\infty} \frac{d \mathbf{k}}{(2 \pi)^{3}} \int_{-\infty}^{\infty} \frac{d \mathbf{q}}{(2 \pi)^{3}} \frac{(\mathbf{q} \cdot \mathbf{v})}{v^{2}} \\
& \times \sum_{\mathbf{R}_{1}, \mathbf{R}_{2}} \sum_{\alpha, \beta, \gamma, \delta} \operatorname{Im}\left[G_{\alpha \gamma}(\mathbf{k})\right] \operatorname{Im}\left[\bar{G}_{\beta \delta}(\mathbf{k})\right] \\
& \times \sum_{\alpha^{\prime}, \beta^{\prime}}\left(S(\mathbf{k})^{-1 / 2}\right)_{\alpha \alpha^{\prime}} I_{\alpha^{\prime} \beta^{\prime}}^{\mathbf{R}_{1}}(\mathbf{q})\left(S(\mathbf{k})^{-1 / 2}\right)_{\beta \beta^{\prime}} \\
& \times \sum_{\gamma^{\prime}, \delta^{\prime}}\left(S\left(\mathbf{k}^{\prime}\right)^{-1 / 2}\right)_{\gamma \gamma^{\prime}} I_{\gamma^{\prime} \delta^{\prime}}^{\mathbf{R}_{2}}(\mathbf{q})\left(S\left(\mathbf{k}^{\prime}\right)^{-1 / 2}\right)_{\delta \delta^{\prime}} \\
& \times e^{i(\mathbf{k}-\mathbf{q}) \cdot\left(\mathbf{R}_{1}-\mathbf{R}_{2}\right)} \delta\left(w_{k k^{\prime}}+\mathbf{q} \cdot \mathbf{v}\right)
\end{aligned}
$$


where

$$
G_{\beta \alpha}(\mathbf{k})=\int_{-\infty}^{E_{F}} \frac{d w}{\pi} G_{\beta \alpha}(\mathbf{k}, w)
$$

and

$$
\bar{G}_{\delta \gamma}\left(\mathbf{k}^{\prime}\right)=\int_{\infty}^{E_{F}} \frac{d w^{\prime}}{\pi} G_{\delta \gamma}\left(\mathbf{k}^{\prime}, w^{\prime}\right)
$$

Equation (57) allows us to calculate the stopping power for He in metals, as a function of the Green-function components $G_{\alpha \beta}(\mathbf{k})$ of the metal (calculated in the orthonormalized basis), using a one-electron hamiltonian $\hat{H}_{0}(\mathbf{k})$ and the overlap matrix $S_{\alpha \beta}^{-1 / 2}(\mathbf{k})$ associated with the atomic wave functions $\psi_{\alpha}$ and $\psi_{\beta}$. Moreover, $\frac{1}{v} \frac{d E}{d x}$ also depends on $I_{\beta \gamma}^{\mathbf{R}}(\mathbf{q})$, the Fourier-transform of the overlap between the atomic orbitals $\psi_{\beta}(\mathbf{r})$ and $\psi_{\gamma}(\mathbf{r}-\mathbf{R})$ as given by Eq. (52) .

On the other hand, in order to analyze the stopping power as a function of $\mathbf{R}$ we take in Eq. (46) $\mathbf{q}^{\prime}=\mathbf{q}-\mathbf{G}$, and only the $\mathbf{G}$ vectors perpendicular to the $\mathbf{v}$ direction. This yields for the $\mathbf{G}$ component of $\frac{1}{v} \frac{d E}{d x}$,

$$
\begin{aligned}
S_{\mathbf{G}} \equiv\left(\frac{1}{v} \frac{d E}{d x}\right)_{\mathbf{G}}= & 4 \pi\left(V_{0}^{\prime}\right)^{2} \int_{-\infty}^{\infty} \frac{d \mathbf{k}}{(2 \pi)^{3}} \int_{-\infty}^{\infty} \frac{d \mathbf{q}}{(2 \pi)^{3}} \frac{(\mathbf{q} \cdot \mathbf{v})}{v^{2}} \Theta\left(k_{F}-k\right) \Theta\left(k^{\prime}-k_{F}\right) \\
& \times I_{k k^{\prime}}(\mathbf{q}) I_{k^{\prime} k}(\mathbf{q}-\mathbf{G}) e^{-i \mathbf{G} \cdot \mathbf{R}} \delta\left(w_{k k^{\prime}}+\mathbf{q} \cdot \mathbf{v}\right),
\end{aligned}
$$

and remember that $\mathbf{k}^{\prime}=\mathbf{k}-\mathbf{q}$.

This equation can be written in a way similar to Eq. (57), as a function of $S(\mathbf{k}), G_{\alpha \beta}(\mathbf{k})$ and,$I_{\alpha \beta}^{\mathbf{R}}$. For the sake of brevity, we shall only mention here that in general the stopping power $S=\frac{1}{v} \frac{d E}{d x}$ can be written as follows:

$$
S_{\mathbf{R}}=S_{0}+\sum_{\mathbf{G}} S_{\mathbf{G}} e^{i \mathbf{G} \cdot \mathbf{R}}
$$

as a function of $\mathbf{R}$, where $S_{0}$ is the mean stopping power given by Eq. (57), and $S_{\mathbf{G}}$ the $\mathbf{G}$ component of Eq. (60). Once we have chosen the $\mathbf{G}$ vectors perpendicular to $\mathbf{v}$, we have calculated $S_{\mathbf{G}}$ by taking an average on the angle between $\mathbf{v}$ and $\mathbf{q}$ as in Eq. (48). 


\section{RESULTS AND DISCUSSIONS}

We have applied the previous formalism to the calculation of the stopping power for He

in alkali metals. For simplicity, the band is assumed to be well described by means of a single $s$ orbital. Then, Eq. (51) can be further simplified into the following equation:

$$
\begin{aligned}
\frac{1}{v} \frac{d E}{d x}= & 2 \pi\left(V_{0}^{\prime}\right)^{2} \int_{-1}^{1} d \cos \theta_{v} \int_{-\infty}^{\infty} \frac{d \mathbf{k}}{(2 \pi)^{3}} \int_{-\infty}^{\infty} \frac{d \mathbf{q}}{(2 \pi)^{3}} \frac{(\mathbf{q} \cdot \mathbf{v})}{v^{2}} \Theta\left(k_{F}-k\right) \Theta\left(k^{\prime}-k_{F}\right) \\
& \times \sum_{\mathbf{R}_{1}, \mathbf{R}_{2}}\left(S(\mathbf{k})^{-1 / 2}\right) I^{\mathbf{R}_{1}}(\mathbf{q})\left(S(\mathbf{k})^{-1 / 2}\right)\left(S\left(\mathbf{k}^{\prime}\right)^{-1 / 2}\right) I^{\mathbf{R}_{2}}(\mathbf{q})\left(S\left(\mathbf{k}^{\prime}\right)^{-1 / 2}\right) \\
& \times e^{i(\mathbf{k}-\mathbf{q})\left(\mathbf{R}_{1}-\mathbf{R}_{2}\right)} \delta\left(w_{k k^{\prime}}+\mathbf{q} \cdot \mathbf{v}\right),
\end{aligned}
$$

or

$$
\begin{aligned}
\frac{1}{v} \frac{d E}{d x}= & 2 \pi\left(V_{0}^{\prime}\right)^{2} \int_{-1}^{1} d \cos \theta_{v} \int_{-\infty}^{\infty} \frac{d \mathbf{k}}{(2 \pi)^{3}} \int_{-\infty}^{\infty} \frac{d \mathbf{q}}{(2 \pi)^{3}} \frac{(\mathbf{q} \cdot \mathbf{v})}{v^{2}} \Theta\left(k_{F}-k\right) \Theta\left(k^{\prime}-k_{F}\right) \\
& \times \sum_{\mathbf{R}_{1}, \mathbf{R}_{2}} S(\mathbf{k})^{-1} S\left(\mathbf{k}^{\prime}\right)^{-1}\left(\sum_{\mathbf{R}_{1}} e^{i(\mathbf{k}-\mathbf{q}) \cdot \mathbf{R}_{1}} I^{\mathbf{R}_{1}}(\mathbf{q})\right)\left(\sum_{\mathbf{R}_{2}} e^{-i(\mathbf{k}-\mathbf{q}) \cdot \mathbf{R}_{2}} I^{\mathbf{R}_{2}}(\mathbf{q})\right) \\
& \times \delta\left(w_{k k^{\prime}}+\mathbf{q} \cdot \mathbf{v}\right),
\end{aligned}
$$

where

$$
I^{\mathbf{R}_{1}}(\mathbf{q})=\int d \mathbf{r} e^{i \mathbf{q} \cdot \mathbf{r}} \psi(\mathbf{r}) \psi\left(\mathbf{r}-\mathbf{R}_{1}\right)
$$

and

$$
S(\mathbf{k})=\sum_{\mathbf{R}} e^{i \mathbf{k} \cdot \mathbf{R}} \int d \mathbf{r} \psi(\mathbf{r}) \psi(\mathbf{r}-\mathbf{R})
$$

Equation (64) can be written in a more symmetric way as follows. Take

$$
\begin{aligned}
I^{\mathbf{R}_{1}}(\mathbf{q}) & =e^{i \mathbf{q} \cdot \mathbf{R}_{1} / 2} \int d \mathbf{r} e^{i \mathbf{q} \cdot\left(\mathbf{r}-\mathbf{R}_{1} / 2\right)} \psi(\mathbf{r}) \psi\left(\mathbf{r}-\mathbf{R}_{1}\right) \\
& =e^{i \mathbf{q} \cdot \mathbf{R}_{1} / 2} \bar{I}^{\mathbf{R}_{1}}(\mathbf{q}),
\end{aligned}
$$

then

$$
\sum_{\mathbf{R}_{1}} e^{i(\mathbf{k}-\mathbf{q}) \cdot \mathbf{R}_{1}} I^{\mathbf{R}_{1}}(\mathbf{q})=\sum_{\mathbf{R}_{1}} e^{i(\mathbf{k}-\mathbf{q} / 2) \cdot \mathbf{R}_{1}} \bar{I} \quad \mathbf{R}_{1}(\mathbf{q})
$$


and

$$
\sum_{\mathbf{R}_{2}} e^{-i(\mathbf{k}-\mathbf{q}) \cdot \mathbf{R}_{2}} I^{\mathbf{R}_{2}}(\mathbf{q})=\sum_{\mathbf{R}_{2}} e^{-i(\mathbf{k}-\mathbf{q} / 2) \cdot \mathbf{R}_{2}} \bar{I} \mathbf{R}_{2}(\mathbf{q})
$$

Equations (62) and (63) yield the stopping power for He as a function of $S(\mathbf{k})$ and $\bar{I} \quad \mathbf{R}(\mathbf{q}) . S(\mathbf{k})$ has been calculated using the atomic wave functions given in Ref. [19]. The calculation of $\bar{I} \quad \mathbf{R}(\mathbf{q})$ is more complicated since the Fouriertransform of the atomic wavefuncions centered on different sites are needed. This is the well-known problem of multicenter integrals. Several solutions have been tried in the literature 20 such as expanding the Slater-type basis in a Gaussian one [21,22]. We have used, however, an adaptative algorithm of integration by Monte Carlo techniques 23] to perform $\bar{I}{ }^{\mathbf{R}}$.

An approximate solution, which yields good results for $S_{0}$, is obtained by replacing

$$
\bar{I}^{\mathbf{R}}(\mathbf{q}) \simeq S(\mathbf{R}) * I(\mathbf{q})
$$

where

$$
S(\mathbf{R})=\int d \mathbf{r} \psi(\mathbf{r}) \psi(\mathbf{r}-\mathbf{R})
$$

and

$$
I(\mathbf{q})=\int d \mathbf{r} e^{i \mathbf{q} \cdot \mathbf{r}} \psi(\mathbf{r}) \psi(\mathbf{r})
$$

Equation (69) is exact in the limit $\mathbf{R}_{i}=0$ or $\mathbf{q} \rightarrow 0$. In general, we expect Eq. (69) to give a good approximation to $I^{\mathbf{R}_{i}}(\mathbf{q})$ if $\mathbf{q} \cdot \mathbf{R}_{i} / 2$ is small.

Introducing Eq. (69) into Eq. (63) yields:

$$
\begin{aligned}
\frac{1}{v} \frac{d E}{d x} & =2 \pi\left(V_{0}^{\prime}\right)^{2} \int_{-1}^{1} d \cos \theta_{v} \int_{-\infty}^{\infty} \frac{d \mathbf{k}}{(2 \pi)^{3}} \int_{-\infty}^{\infty} \frac{d \mathbf{q}}{(2 \pi)^{3}} \frac{(\mathbf{q} \cdot \mathbf{v})}{v^{2}} \Theta\left(k_{F}-k\right) \Theta\left(k^{\prime}-k_{F}\right) \\
& \times \frac{I(q)}{S(\mathbf{k})} \frac{I(q)}{S\left(\mathbf{k}^{\prime}\right)}\left|\sum_{\mathbf{R}} S(\mathbf{R}) e^{i(\mathbf{k}-\mathbf{q} / 2) \cdot \mathbf{R}}\right|^{2} \delta\left(w_{k k^{\prime}}+\mathbf{q} \cdot \mathbf{v}\right) .
\end{aligned}
$$

Equation (72) is the basis of our approximation to Eqs. (62) and (63). We should also mention that $E_{k}$ (the electron energy band of the alkali metal) has been assumed to follow a free electron dispersion law. 
Before discussing the numerical results given by Eq. (72), it is worth considering the results obtained by neglecting all the overlaps between the alkali atom wavefunctions. Then we write

$$
S(\mathbf{R})= \begin{cases}1, & \mathbf{R}=0 \\ 0, & \mathbf{R} \neq 0\end{cases}
$$

and

$$
S(\mathbf{k})=1
$$

and replace Eq. ( $\sqrt{72})$ by the following equation:

$$
\begin{aligned}
\frac{1}{v} \frac{d E}{d x} & =2 \pi\left(V_{o}^{\prime}\right)^{2} \int_{-1}^{1} d \cos \theta_{v} \int_{-\infty}^{\infty} \frac{d \mathbf{k}}{(2 \pi)^{3}} \int_{-\infty}^{\infty} \frac{d \mathbf{q}}{(2 \pi)^{3}} \frac{(\mathbf{q} \cdot \mathbf{v})}{v^{2}} \Theta\left(k_{F}-k\right) \Theta\left(k^{\prime}-k_{F}\right) \\
& \times\left|\int d \mathbf{r} \psi^{2}(\mathbf{r}) e^{i \mathbf{q} \cdot \mathbf{r}}\right|^{2} \delta\left(w_{k k^{\prime}}+\mathbf{q} \cdot \mathbf{v}\right) .
\end{aligned}
$$

It is also convenient to discuss at this point the stopping power given by the following simple model: a uniform electron gas interacting with a slowly moving He atom by means of the following contact potential

$$
\hat{H}_{\text {pert }}=V_{0}^{\prime} \delta(\mathbf{r}-\mathbf{v} t)
$$

Here $V_{0}^{\prime}$ is assumed to be the same local potential introduced in Eq. (44). It is an easy task to develop this model following the same steps as discussed above for the LCAO approach and find the following expression for the stopping power

$$
\frac{1}{v} \frac{d E}{d x}=4 \pi\left(V_{0}^{\prime}\right)^{2} \int_{-\infty}^{\infty} \frac{d \mathbf{k}}{(2 \pi)^{3}} \int_{-\infty}^{\infty} \frac{d \mathbf{q}}{(2 \pi)^{3}} \frac{(\mathbf{q} \cdot \mathbf{v})}{v^{2}} \Theta\left(k_{F}-k\right) \Theta\left(k^{\prime}-k_{F}\right) \delta\left(w_{k k^{\prime}}+\mathbf{q} \cdot \mathbf{v}\right)
$$

The integral in $\cos \theta_{v}$ equals 2 because in the latter expression $\frac{1}{v} \frac{d E}{d x}$ depends only on $|\mathbf{v}|$.

Comparing Eqs. (75) and (77), we see that their only difference is associated with the term $|I(q)|^{2}=\left|\int d \mathbf{r} \psi^{2}(\mathbf{r}) e^{i \mathbf{q} \cdot \mathbf{r}}\right|^{2}$, which gives the form factor of the metal orbital. We should also comment, regarding Eq. (72) that in the alkali metals $S(\mathbf{k}) \sim S\left(k_{F}\right)$ since, in the low velocity limit we are considering $\mathbf{k}$ and $\mathbf{k}^{\prime}$ are located near the Fermi sphere, $S(\mathbf{k})$ 
being almost constant on this surface that presents a very small anisotropy. Then, Eq. (72) can be obtained from Eq. (75) by replacing the form factor $I(\mathbf{q})$ by

$$
D(\mathbf{q}, \mathbf{k})=\frac{I(q) S(\mathbf{k}-\mathbf{q} / 2)}{S(\mathbf{k})},
$$

where

$$
S(\mathbf{k}-\mathbf{q} / 2)=\sum_{\mathbf{R}} S(\mathbf{R}) e^{i(\mathbf{k}-\mathbf{q} / 2) \cdot \mathbf{R}} .
$$

Thus the three different cases we are considering yield the same equation for the stopping power, but for a specific factor taking the values $1, I(q)$ and $D(\mathbf{q}, \mathbf{k})$, for the free-electron gas (FEG), the LCAO model with $S(\mathbf{R})=0$ for $\mathbf{R} \neq 0$ (LCAO-I), and the LCAO model with $S(\mathbf{R}) \neq 0$ (LCAO-II), respectively. What is of interest to realize about this discussion is that the free-electron-gas model overestimates the stopping power, while the simplest LCAO model underestimates it. In Table [, we give the three values of the mean stopping power $S_{0}$, for $\mathrm{He}$ in $\mathrm{Na}$ as calculated from these equations. As shown in this Table $\llbracket$ the free electron gas model yields a stopping power three times too large, while in the LCAO-I model $\frac{1}{v} \frac{d E}{d x}$ is about eight times too small.

One word of caution must be put here. The FEG model discussed here can not be compared directly with the LDA used to calculate the stopping power of He in metals. The point to notice is that in the model defined by Eq. (86), $V_{0}^{\prime}$ is the contact potential for the interaction of He with the $s$ orbitals of the alkali-metal atoms. The model of Eq. (76) is only introduced here in order to explain how the form factor of Eqs. (78) or (75) is the main term controlling the He stopping power.

As regards the factor $D(\mathbf{q}, \mathbf{k})$ used to calculate $\frac{1}{v} \frac{d E}{d x}$ in the LCAO-II approximation, notice the strong dependence that $D(\mathbf{q}, \mathbf{k})$ has on the number of neighbors used to calculate $S(\mathbf{k})=\sum_{\mathbf{R}} S(\mathbf{R}) e^{i \mathbf{k} \cdot \mathbf{R}}$ and $S(\mathbf{k}-\mathbf{q} / 2)$ in Eq. (78). We have found that in order to get a reasonable accuracy (around 5\%) it is necessary to add up to the fifth or sixth neighbor, depending on the alkali metal.

As mentioned above, Eq. (63) has been accurately calculated for Na using Monte Carlo techniques. We have found that this Monte Carlo calculation yields 


$$
\frac{1}{v} \frac{d E}{d x}=0.085 \text { a.u. }(\mathrm{Na})
$$

a value a little larger than the one found using our LCAO-II approximation. By assuming

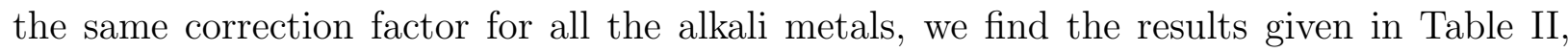
column (a). This Table also shows the theoretical figures obtained by Echenique, Nieminen, and Ritchie [11].

We see from Table $\mathbb{1}$, column (a), that the results for $\mathrm{K}$ and $\mathrm{Rb}$ are in excellent agreement with Ref. [11], although the stopping powers we find for Li and $\mathrm{Na}$ are a little larger. This difference can be partially attributed to the simple model we are using, since a single $s$ orbital per alkali-metal atom has been assumed to form the metal conduction band. This approximation can be expected to be a reasonable one for very electropositive atoms like $\mathrm{K}$ and Rb, but not so appropriate at least for Li. Thus, in the calculations of Papaconstantopoulos 24] for Li, only $52 \%$ of the occupied density of states has a $s$ like character. If we introduce in the results of Table II, a factor

$$
n_{s}^{2} / n_{s}^{2}(\mathrm{Rb})
$$

which normalizes the stopping power of each alkali metal to the total number of $s$ electrons with respect to $\mathrm{Rb}$, we find the results of Table $\mathbb{1}$, column (b), in much better agreement with the LDA calculations.

The conclusion we can draw from these results is that the method developed in this paper is quite appropriate to calculate the stopping power for He moving slowly in alkali metals. We can also expect that the method will be useful to calculate stopping powers for atoms in transition metals.

In a further step we have calculated, using Monte Carlo techniques, the stopping power dependence on the ion position (for He moving in a channeled direction). We have considered that He moves in a Na crystal along the [100] direction. We have calculated the different $\mathbf{G}$ reciprocal vectors contributing to the stopping power [Eq. (60]]; this implies taking the $\mathbf{G}$ vectors perpendicular to the [100] direction. In a bcc lattice, the first reciprocal vectors to 
be considered are the followings: $\mathbf{G} \equiv \frac{2 \pi}{a}(0,1,1), \frac{2 \pi}{a}(0,0,2)$ etc. Using Eqs. (60) and (61) we have obtained the stopping power Fourier components $S_{G}$ shown in Table III].

Figure 3 shows $S_{\mathbf{R}}$, with $\mathbf{R}$ changing in a [100] plane. The main conclusion we can draw from these calculations is the strong dependence that the stopping power shows as a function of the impact parameter: the stopping power can vary as much as $100 \%$ for different impact distances. We should comment that these changes are not associated with the electronic metal charge [13]; this charge, as obtained in our LCAO approach with an $s$ level per atom, appears to be almost constant in the crystal lattice except very close to the atomic sites. For a He-atom channeled along the $\mathrm{Na}$ [100] direction, one expects some kind of oscillatory motion of the atom, with the impact parameter changing along the He trajectory. Then, the mean-stopping power for the channeled case would appear as an average of the different values shown in Fig. 3 around the minimum value of the stopping power. Each case should be analyzed specifically, but assuming the incoming atom to explore only half of the total available space, one would get around $50-60 \%$ of $S_{0}$, namely 0.04 a.u., $80 \%$ of the value calculated in LDA.

\section{CONCLUSIONS}

The aim of this work has been to develop a first-principles, free-parameter, approach based on a LCAO method to calculate the stopping power for atoms moving in condensed matter. In the past few years the interest in, generally speaking, tight-binding methods 25] for analyzing the electronic properties of solids has increased a lot. This emphasis is partially due to the interest in using a local point of view, closely related to the chemistry of the local environment. The work presented in this paper follows this general trend and tries to apply the ideas recently developed in Refs. [14,15] for analyzing the electronic properties of solids following a LCAO method, to the stopping power area. In the long term, this approach can be expected to be also useful for analyzing other dynamical processes like the charge transfer between moving ions and the solid, sticking mechanisms, etc. 
In Sec. II, we have presented our general approach and have related the stopping power for atoms, in the low-velocity limit, to the electronic properties of the crystal as described using a LCAO method. All the parameters appearing in Eq. (9), the general equation giving the stopping power, can be obtained from the local wave funcions of the atoms forming the crystal. Equation (91) has been applied to the case of He moving in alkali metals. He is a simple atom, but the alkali metals present a strong test to our method as their atomic wave funcions interact strongly with each other up to large separations. In Sec. IV, we have presented our results and have found that the stopping power for He is very well described with our local LCAO approach, if the interaction between different alkali metal atomic orbitals is included, at least, up to fifth neighbor.

We conclude that the LCAO method discussed in this paper offers the possibility of calculating accurately the stopping power for ions moving in solids. This could be a convenient framework for analyzing solids having localized $d$ bands and for discussing specific geometries like the case of atoms moving near surfaces, or the channeled case discussed in Sec. V.

\section{ACKNOWLEDGMENTS}

This work has been partially funded by the Spanish CICYT under contract no. PB89165. One of the authors (J.J.D.) thanks Ministerio de Educación y Ciencia and Universidad Autónoma de Madrid, Spain, for their financial support. F.F. acknowledges support by Iberdrola S.A. We thank R. Ritchie, N. Lorente, P.M. Echenique, and M. Jakas for helpful discussion. 


\section{REFERENCES}

[1] E. Rutheford, Philos Mag. 21, 669 (1911).

N. Bohr, ibid. 25,16 (1913).

[2] H.A. Bethe, Ann. Phys. (Leipzig) 5, 325 (1930).

[3] E. Fermi, Z. Phys. 29, 315 (1927).

[4] E.J. Williams, Rev. Mod. Phys. 17, 217 (1945).

[5] J. Lindhard, K. Dans, Mat. Fys. Met. 28, 8 (1954).

[6] W. Brant, in Atomic Collisions in Solids , edited by S. Datz, B.R. Appelton, and C.D. Moak (Plenum, New York, 1975).

[7] R.H. Ritchie and J.C. Ashley, J. Phys. Chem. Solids 26 , 1689 (1965).

[8] A. Arnau, M. Peñalba, P.M. Echenique, F. Flores, and R.H. Ritchie, Phy. Rev. Lett. 65, 1024 (1990).

[9] F. Flores, in Interaction of Charged Particles with Solids and Surfaces, edited by A. Gras-Marti et al. (Plenum, New York, 1991).

[10] P.M. Echenique, F. Flores, and R.H. Ritchie, Solid State Physics Series 43, 229 (1990).

[11] P.M. Echenique, R.M. Nieminen, and R.H. Ritchie, Solid State Commun. 37, 779 (1981).

[12] P. Bauer, F. Kastner, A. Arnau, A. Salin, P.D. Fainstein, V.H. Ponce, and P.M. Echenique, Phys. Rev. Lett. 69, 1137 (1992).

[13] P.L. Grande and G. Schwietz, Phys. Lett. A 163, 439 (1992).

[14] E.C. Goldberg, A. Martin-Rodero, R. Monreal, and F. Flores, Phys Rev. B 39,5684 (1989).

[15] F.J. García Vidal, A. Martin-Rodero, F. Flores, J. Ortega, and R. Perez , Phys. Rev. 
B 44, 11412 (1991).

[16] J.R. Sabin, J. Oddershede, and G.H.F. Diercksen, Phys. Rev. A 42, 1302 (1990).

D.E. Meltzer, J.R. Sabin, and S.B. Trickey, ibidi. 41, 220 (1990).

[17] P.O. Löwdin, J. Chem. Phys. 18, 365 (1950).

[18] F. Sols, Ph.D. Thesis, Universidad Autónoma de Madrid (1985).

F. Sols and F. Flores, Solid State Commun. 42, 687 (1982).

[19] E. Clementi and C. Roeti, Atomic Data and Nuclear Tables 14, 177 (1974).

[20] H. Wormeester, H.J. Borg, and A. Van Silfhout, Surf. Sci. 258, 197 (1991).

[21] E.H. Morknersen, J. Oddershede and J.R. Sabin, Nucl. Instrum. Methods Phys. Rev. (to be published).

[22] S.F. Boys, Proc. R. Soc. London Ser A 200, 542 (1950).

[23] G. Peter Lepage, J. of Comput. Phys. 27, 192 (1978).

[24] D.A. Papaconstantopoulos, in Handbook of Band Structure of the Elemental Solids (Plenum, New York, 1986).

[25] W. A. Harrison, in Electronic Structure and Properties of Solids (Freeman, San Francisco, 1980). A.D. Sutton, M.W. Finnis, D.G. Pettiford, and Y. Ohta, J. Phys. C 21, 35 (1988); J.A. Majewshi, and P. Vogl, Phys. Rev. Lett. 57, 1366 (1986). 


\section{FIGURES}

FIG. 1. He moving inside a metal.

FIG. 2. Schematic representation of a He atom interacting with a metal band simulated by a metal level $E_{M}$.

FIG. 3. Stopping power for He moving inside a Na crystal along the [100]-direction. The stopping power is normalized with respect to its mean value. The coordinates correspond to a (100) plane perpendicular to the projectile trajectory. The atomic rows along the [100] direction are projected onto the points having the coordinates: $(0,0),(0,1),(1,1),(1,0)$ and, $(0.5,0.5)$. 


\section{TABLES}

\begin{tabular}{llll}
\hline \hline & FEG & LCAO-I & LCAO-II \\
\hline$\frac{1}{v} \frac{d E}{d x}$ (a.u.) & 0.140 & 0.007 & 0.056 \\
\hline \hline
\end{tabular}

TABLE I. Stopping power for He in $\mathrm{Na}$ as calculated for the free electron model (FEG), the

LCAO model neglecting the metal wave functions overlaps (LCAO-I), and the LCAO model taking into account these overlaps (LCAO-II).

\begin{tabular}{llll}
\hline \hline & $(\mathrm{a})$ & $(\mathrm{b})$ & $(\mathrm{c})$ \\
\hline $\mathrm{Li}$ & 0.260 & 0.110 & 0.100 \\
$\mathrm{Na}$ & 0.085 & 0.068 & 0.053 \\
$\mathrm{~K}$ & 0.026 & 0.023 & 0.023 \\
$\mathrm{Rb}$ & 0.015 & 0.015 & 0.016 \\
\hline \hline
\end{tabular}

TABLE II. Stopping power in a.u. for He in different alkali metals. (a) Our results, as calculated using Monte Carlo techniques. (b) Our results with the $s$ ocupancy correction. (c) ENR, from Ref. [11.

\begin{tabular}{lr}
\hline \hline & atomic units \\
\hline $\mathrm{S}(0,0,0)$ & $0.85 \times 10^{-1}$ \\
$\mathrm{~S}(0,1,1)$ & $0.18 \times 10^{-1}$ \\
$\mathrm{~S}(0,0,2)$ & $-0.04 \times 10^{-1}$ \\
\hline \hline
\end{tabular}

TABLE III. Stopping Power for He in Na for the channeled direction [100] 